\title{
Uma Abordagem para Definição de Processos Baseada em Reutilização Visando à Alta Maturidade em Processos
}

\author{
Ahilton Silva Barreto ${ }^{1}$, Leonardo Gresta Paulino Murta ${ }^{2}$, Ana Regina Rocha ${ }^{1}$ \\ ${ }^{1}$ COPPE/UFRJ - Universidade Federal do Rio de Janeiro \\ Caixa Postal 68511 - CEP: 21945-970 - Rio de Janeiro - RJ \\ ${ }^{2}$ Instituto de Computação - Universidade Federal Fluminense (UFF) \\ Niterói - RJ \\ ahilton@cos.ufrj.br, leomurta@ic.uff.br, darocha@cos.ufrj.br
}

\begin{abstract}
This work presents a reuse-based approach to define software processes, which also considers requirements from the higher maturity context. Techniques usually applied on software product development are adapted to software processes definition. Furthermore, information on subprocesses stability, performance and capacity are used throughout process definition. The proposed approach also comprises different strategies to define processes for and with reuse and a set of supporting tools. To assess the viability of the approach, experimental evaluations were performed and their results indicate that the use of the proposed approach is possible and provide benefits to processes definition.
\end{abstract}

Resumo. Este trabalho apresenta uma abordagem para definição de processos baseada em reutilização, que considera, também, a definição de processos em alta maturidade. Técnicas de reutilização normalmente aplicadas no desenvolvimento de produtos de software são adaptadas para a definição de processos de software. Informações sobre estabilidade, desempenho e capacidade dos subprocessos são utilizadas ao longo da definição de processos. A abordagem inclui estratégias para definição de processos para e com reutilização e um conjunto de ferramentas de apoio. Para avaliar a viabilidade das propostas deste trabalho, foram realizadas avaliações, cujos resultados fornecem indícios de que o uso da abordagem proposta é viável e fornece bons resultados para a definição de processos.

\section{Introdução}

Definir um processo de software não é uma atividade simples; exige experiência e envolve o conhecimento de muitos aspectos da engenharia de software. Assim, a atividade normalmente exige um profissional especializado que consiga harmonizar todos os fatores envolvidos.

No contexto de organizações que buscam a alta maturidade para seus processos, a definição de processos se torna ainda mais complexa. Modelos de maturidade e normas como o CMMI-DEV (Sei, 2010), o MPS.BR (Softex, 2011) e a ISO/IEC 15504 (Iso/Iec-15504, 2004) estabelecem que, em organizações de maior maturidade, os processos devem ser definidos com base em unidades de processo menores, 
normalmente chamadas de subprocessos ou elementos de processos. Estabelecem, ainda, que os processos devem ser definidos com base na seleção dos subprocessos mais adequados para compor o processo com base na sua estabilidade histórica, em dados de capacidade e desempenho dos subprocessos, além de outros critérios previamente estabelecidos para os projetos. Devem ser consideradas diferentes alternativas de processos e subprocessos, e o desempenho dos subprocessos deve ser considerado em relação aos objetivos de qualidade e desempenho dos projetos.

Como forma de promover a reutilização do conhecimento relacionado a processos de software, técnicas de reutilização têm sido adaptadas do desenvolvimento de produtos de software para o contexto da definição de processos de software (Reis, 2002; Rombach, 2005; Washizaki, 2006; Aleixo et al., 2010). O propósito é facilitar a definição de processos, diminuindo o custo e o esforço associado à atividade, além de possivelmente aumentar a qualidade dos processos gerados, inclusive tornando a realização da atividade acessível a profissionais menos experientes. Ou seja, espera-se que com a aplicação de técnicas de reutilização seja possível obter no contexto de processos benefícios semelhantes àqueles obtidos pelo desenvolvimento de produtos a partir da aplicação dessas técnicas. Assim, conceitos como componentes, arquiteturas, linhas de produtos e padrões têm sido utilizados para a definição e melhoria de processos de software. A utilização dessas técnicas pode favorecer o aproveitamento das oportunidades de reutilização de processos e também auxiliar na definição de processos com base na seleção de subprocessos e de alternativas de processo mais adequadas.

Neste contexto, o objetivo deste trabalho consiste em definir uma abordagem para apoiar a definição de processos de software baseada em técnicas de reutilização, considerando requisitos da definição de processos em alta maturidade. A abordagem se propõe a tornar a definição de processos de software mais simples e eficiente, por meio do aproveitamento das oportunidades de reutilização existentes nos diferentes contextos em que processos precisam ser definidos, além de possibilitar que informações como medidas, estabilidade e desempenho dos subprocessos sejam consideradas na definição. Assim, o conhecimento de engenheiros de processo mais experientes e o conhecimento obtido através do uso dos processos e de seus componentes poderá ser mais facilmente reutilizado na definição de processos de software.

O objetivo geral do trabalho pode ser decomposto nos seguintes objetivos específicos: (i) definir uma adaptação dos conceitos de reutilização de software para o contexto de processos de software; (ii) definir estratégias de definição de processos para e com reutilização; (iii) definir uma estratégia de utilização da abordagem em diferentes contextos (instituições implementadoras, organizações, projetos); (iv) definir e desenvolver ferramentas de apoio à execução da abordagem; e (v) avaliar a abordagem proposta em relação a fatores como: esforço para definir processos, aderência dos processos definidos, benefícios e dificuldades esperadas e aderência à alta maturidade.

Este trabalho está organizado em 5 seções, incluindo esta introdução. A seção 2 apresenta uma breve revisão da literatura sobre os temas principais do trabalho, incluindo a descrição de um estudo baseado em revisão sistemática. Na seção 3 é apresentada a abordagem proposta, seguida de sua avaliação, apresentada na seção 4. A seção 5 conclui o trabalho apresentando contribuições, limitações e perspectivas futuras. 


\section{Definição e Reutilização de Processos de Software}

O processo de software é um fator crítico para o desenvolvimento de produtos de software de qualidade, uma vez que tem por objetivo gerenciar e transformar as necessidades dos usuários em um produto de software que atenda a essas necessidades. (Acuña et al., 2000). Dada a importância do tema, diversas normas e modelos de maturidade (Iso/Iec-15504, 2004; Sei, 2010; Softex, 2011) têm se preocupado em definir requisitos e guias a serem seguidos por uma organização que deseje definir seus processos. Mais ainda, a definição de processos de software tem sido considerada requisito fundamental para que uma organização atinja níveis mais altos de maturidade.

Segundo Card et al. (2008), é possível resumir o objetivo geral da alta maturidade como sendo "gerenciar o desempenho dos processos de forma que produzam os resultados desejados de maneira confiável". Esse objetivo pode ser desdobrado em três outros: (i) tornar o processo estável, ou seja, eliminar causas de variação e comportamento incomum para atingir desempenho consistente; (ii) gerenciar o processo para atingir os objetivos, ou seja, estabelecer objetivos, predizer o desempenho futuro e ajustar o desempenho para atingir os objetivos; e (iii) identificar melhorias no processo, ou seja, eliminar causas de variação e obstáculos para o desempenho, ou implementar métodos melhores para alinhar o desempenho com as expectativas do cliente.

Conforme mencionado na seção 1 , no contexto da alta maturidade os modelos de maturidade estabelecem requisitos adicionais para a realização da definição de processos. No CMMI-DEV (Sei, 2010), a área Gerência Quantitativa de Projetos define a prática específica "Compor o Processo Definido", a qual determina que, usando técnicas estatísticas ou outras técnicas quantitativas, deve-se compor o processo definido que permite que o projeto alcance seus objetivos de qualidade e desempenho de processo. Compor o processo definido do projeto vai além da seleção e adaptação de processo. Envolve, também, a identificação de alternativas para um ou mais processos ou subprocessos, a realização de análise quantitativa do desempenho e a seleção das alternativas que são mais capazes de auxiliar o projeto a atingir seus objetivos de qualidade e desempenho de processo (Sei, 2010).

Há diversos trabalhos na literatura que defendem que processos têm semelhanças com software, e que é possível aplicar métodos e técnicas de desenvolvimento de software na definição de processos (Osterweil, 1987). Da mesma forma que há muitas analogias entre processos e produtos de software, podem ser feitas também analogias interessantes entre reutilização de processos de software e reutilização de produtos de software. Assim, muitas técnicas da reutilização de software tradicional, tais como a utilização de componentes, arquiteturas e linhas de produtos têm sido aplicadas também no contexto de processos de software.

Um componente de processo pode ser considerado o encapsulamento de informações e comportamentos de processo em um dado nível de granularidade (Gary e Lindquist, 1999). Já uma arquitetura de processos pode ser definida como a ordenação, interfaces, interdependências, e outros relacionamentos entre os elementos de processo (Sei, 2010). Uma linha de processos pode ser considerada uma linha de produtos cujos produtos são processos de software (Rombach, 2005). As linhas de processo modelam semelhanças e variabilidades em um conjunto de processos. Semelhanças são representadas pelo processo central, que é formado por partes comuns de um conjunto 
de processos. Variabilidade é representada pelos pontos de variação e variantes do processo. Pontos de variação são componentes que podem ser modificados de acordo com as características de um utilização específica. Variantes de processo são os componentes concretos candidatos que são aplicados nos pontos de variação.

Devido à relevância do tema reutilização de processos de software, foi realizada, neste trabalho, uma revisão disciplinada da literatura através da execução de um estudo baseado em revisão sistemática (Montoni, 2007), cuja questão de pesquisa principal foi: como técnicas de reutilização são utilizadas para apoiar a definição de processos de software. Foram definidas, também, questões secundárias, para auxiliar na resposta da questão principal. São elas: (QS1) Quais técnicas de reutilização são usadas? (QS2) Qual apoio ferramental é oferecido? (QS3) Como o contexto multiorganizacional é tratado? (QS4) Como são considerados os dados de execução dos processos na definição de processos? (QS5) Como se apoia a realização da definição de processos conforme práticas de alta maturidade? (QS6) Como se avaliou a proposta descrita? O objetivo do estudo foi analisar relatos de experiência e publicações científicas sobre definição de processos de software, com o propósito de caracterizar abordagens (ex.: técnicas, métodos, processos, ferramentas), com relação à utilização de técnicas de reutilização, do ponto de vista de pesquisadores, no contexto industrial e acadêmico.

De um total de 301 artigos (identificados nas bibliotecas digitais da IEEE, Scopus e Compendex e nos anais do SBQS), apenas 57 atenderam a todos os filtros e critérios definidos. Com base nas questões secundárias do estudo baseado em revisão sistemática realizado, foi possível perceber que: (i) não há um consenso sobre as características das técnicas utilizadas, uma vez que várias definições diferentes existem para cada uma delas e muitas vezes ocorrem sobreposições; (ii) das publicações analisadas, um grande percentual não descreve apoio ferramental (53\%) e a maioria não menciona a utilização de dados da execução para apoiar na definição dos processos (77\%); (iii) mais da metade das publicações analisadas (54\%) não mencionam qualquer avaliação da abordagem que propõem ou fornecem apenas exemplos; (iv) o contexto multiorganizacional é considerado em pouquíssimos trabalhos (12\%), indicando que as oportunidades de reutilização neste contexto ainda merecem melhor aproveitamento; (v) a definição de processos em alta maturidade não é mencionada nas abordagens citadas, apesar de algumas características dessas abordagens poderem auxiliar no caminho para a alta maturidade, mas ainda há muita necessidade de apoio específico nesse contexto.

As considerações expostas parecem mostrar que ainda é necessário um amadurecimento maior do tema. Boa parte dos trabalhos citados parece relatar apenas ideias, dado o grande número de propostas sem mencionar apoio ferramental ou qualquer utilização. Assim, percebe-se a necessidade de mais trabalhos de forma a se criar massa crítica sobre o assunto, principalmente no que se refere à alta maturidade.

\section{Abordagem Proposta}

A abordagem proposta neste trabalho deveria atender aos seguintes requisitos: (i) possuir uma adaptação dos conceitos relevantes da reutilização de produtos de software para o contexto da definição de processos de software; (ii) possuir estratégias de definição de processos para e com reutilização; (iii) permitir a definição de processos para projetos considerando requisitos de alta maturidade; (iv) possuir uma estratégia 
para aplicação da abordagem em diferentes contextos; e (v) possuir ferramental de apoio. Esses requisitos foram definidos com base na pesquisa na literatura, que possibilitou um entendimento sobre as principais questões envolvidas na definição de processos, sobre como essas questões têm sido tratadas pela academia e pela indústria e sobre oportunidades de pesquisa existentes. A experiência da COPPE/UFRJ na definição, implantação e melhoria de processos também auxiliou na determinação dos requisitos.

\subsection{Visão Geral e Fundamentos da Abordagem Proposta}

Para possibilitar a reutilização de processos de software foi necessário, primeiramente, definir como o conhecimento relacionado aos processos de software deveria ser estruturado e representado de forma a permitir e facilitar sua posterior reutilização, definindo, também, a semântica de cada conceito envolvido. Optou-se por construir o modelo conceitual deste trabalho a partir de ontologias existentes relacionadas ao tema do trabalho. No entanto, diversos conceitos e relações necessários ao trabalho, principalmente relacionados à reutilização, não são considerados nas ontologias existentes. Com isso, classes, atributos e associações requeridos, que representam elementos que não são necessariamente consensuais no domínio, mas que são fundamentais para este trabalho, estão também presentes no modelo. O modelo definido foi dividido em quatro seções principais:

- Estrutura Central de Reutilização de Processos: define os elementos principais da abordagem, incluindo: Elemento de Processo (Encapsulamento de informações e comportamentos de processo em um dado nível de granularidade); Atividade (Elemento de processo completamente definido que não será reutilizado diretamente); Arquitetura de Processos ( "esqueleto" que o processo deve possuir, seus principais elementos e relações, sem necessariamente detalhar os elementos); Linha de Processos (arquitetura independente que modela variabilidades e semelhanças entre processos e podem definir pontos de variação ou opcionalidades); e Componente de Processo (detalhado a seguir).

- Características de Processo - descreve as características de processo e suas relações. Uma característica de processo pode ser vista como um aspecto, qualidade ou caracterização com a qual o processo precisa ser compatível. As características restringem a utilização de componentes, estabelecendo um conjunto de regras que guiam a definição do processo. Podem se relacionar entre si por meio de relacionamentos de dependência e conflito. Podem ser associadas a componentes ou linhas de processo.

- Medição e Comportamento de Processos - define os conceitos principais relacionados ao tema e estabelece que componentes de processo podem ser medidos, podem ser associados a baselines de desempenho e podem ser associados a objetivos de qualidade e desempenho de processos. Essa seção é muito importante para o atendimento dos requisitos da alta maturidade.

- Processos Padrão, Processos Definidos, Projetos e Derivações - além de definir esses conceitos, estabelece que processos são definidos a partir de linhas de processos ou de outros processos. As derivações indicam o que mudou em um processo em relação à linha ou processo que o originou. 
O conceito de componente de processo merece destaque nesta abordagem. É a unidade básica de definição de processos com reutilização nesta abordagem e possui algumas características importantes: (i) é relevante para ser reutilizado; (ii) representa um subprocesso relevante de um processo de mais alto nível, que pode ser realizado de uma ou de várias maneiras; (iii) é relevante para ser medido e ter sua estabilidade e desempenho analisados; (iv) pode atuar como um container que encapsula outros elementos de processo; (v) admite variabilidades, ou seja, pode representar diferentes maneiras de realizar uma parte de um processo. Componentes de processo podem ser concretos ou abstratos. Componentes concretos não admitem qualquer variabilidade e precisam ser executados da forma que estão descritos. Já os componentes abstratos admitem variabilidades, não estando vinculado a uma única forma de realização.

Uma vez definida a modelagem dos conceitos relacionados à reutilização de processos de software usada neste trabalho, foi possível determinar os principais cenários de uso para a abordagem (Barreto et al., 2011). Nesta abordagem, considera-se que a reutilização de processos de software pode ocorrer em diferentes contextos e níveis de abrangência: (i) Instituições implementadoras de processo (IIs), que podem necessitar definir processos padrão semelhantes para um conjunto de organizações diferentes; (ii) Organizações de Software, que podem necessitar definir seus processos padrão, ou especializar esses processos para situações comuns na organização. Uma biblioteca de componentes reutilizáveis de processos de software poderia auxiliar nas definições tanto de processos padrão como na definição dos processos definidos dos projetos; (iii) Projetos de Software que tenham tido seu processo definido a partir de uma biblioteca de componentes reutilizáveis de processos de software contribui para essa biblioteca através da coleta de informações de uso, medidas, solicitações de melhoria, entre outras informações relacionadas à execução dos componentes de processo. A Figura 1 ilustra a reutilização de processos nas IIs.

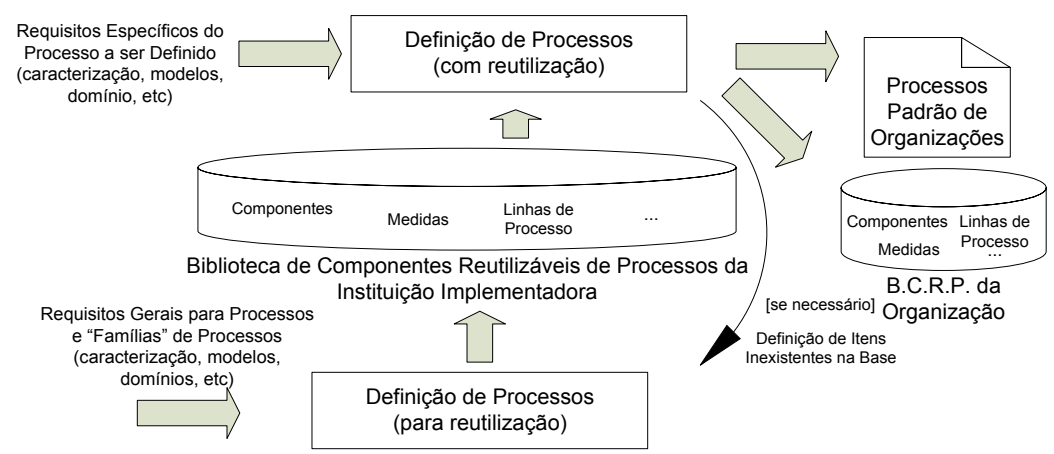

Figura 1. Reutilização de processos em Ils

Após a definição dos principais conceitos da abordagem e de seus principais cenários de uso, se fez necessário entender melhor e obter mais informações sobre a reutilização de processos de software, de modo a direcionar os passos seguintes da pesquisa. Assim, uma vez que os usuários potenciais da abordagem definida são engenheiros de processo, era importante capturar suas expectativas a respeito dos benefícios e dificuldades relacionados aos principais conceitos de reutilização de processos sendo utilizados neste trabalho. Além disso, se processos de software são software também, como enunciado por Osterweil (1987), era de se esperar que os benefícios e as dificuldades esperados a partir da reutilização de processos e a partir da 
reutilização de produtos de software fossem similares. Assim, com o objetivo de capturar as opiniões de engenheiros de processo, um subconjunto dos principais benefícios e dificuldades relacionados a iniciativas de reutilização de produtos de software foi adaptado para o contexto de processos de software e considerado. Para obter as opiniões necessárias e também verificar as similaridades entre reutilização de processos e de produtos de software, foi planejada e executada uma pesquisa de opinião (survey) (Barreto et al., 2011). A pesquisa pedia aos participantes que avaliassem os benefícios e dificuldades esperados a partir da aplicação das abordagens de reutilização. Participaram da pesquisa um total de 23 participantes, todos com boa experiência em definição de processos tanto na indústria como na academia. Os resultados da pesquisa indicaram que linhas de Processo representam a abordagem da qual são esperados mais benefícios, que os benefícios mais esperados são: "Melhor produtividade" e "Diminuição de retrabalho", que são esperadas dificuldades em intensidade menor que benefícios e que "Apoio ferramental inadequado" foi a dificuldade esperada em maior intensidade. Assim, consolidando os resultados da pesquisa foi possível perceber que linhas de processo tendem a trazer mais benefícios, desde que com apoio ferramental adequado. Esse resultado foi importante para definir o foco do trabalho em linhas de processo e no desenvolvimento de ferramentas de apoio.

\subsection{Definindo Processos para Reutilização}

Neste trabalho a estratégia para definir processos para reutilização envolve, tanto no contexto de instituições implementadoras como no contexto de organizações os seguintes passos genéricos: (i) Definição de características de processo; (ii) Definição de relacionamentos entre características de processo; (iii) Definição de componentes de processo; (iv) Associação entre características de processo e componentes de processo; (v) Definição de linhas de processo, utilizando características e componentes de processo e modelando as semelhanças e variabilidades entre processos; (vi) Avaliação e aprovação das características, componentes e linhas de processo definidas.

$\mathrm{Na}$ definição de processos para reutilização pode ser adotada uma abordagem bottom-up ou top-down (ou ainda uma combinação entre elas). A abordagem top-down começa com a definição de características de processo que são usadas como ponto de partida para a definição de componentes e linhas de processo derivadas dsas características. Ou seja, deve ser usada quando há um conjunto bem definido de requisitos para o processo e não há processos legados. Já na abordagem bottom-up, a definição se inicia pela definição de componentes de processo, baseados em processos previamente definidos (legados). Esses componentes são, então, caracterizados e conectados até que se chegue ao conjunto completo com características, componentes e linhas. Ou seja, funciona como uma reengenharia de processos. A Figura 2 ilustra os passos das abordagens bottom-up e top-down para definir processos para reutilização.

O detalhamento das abordagens fornece orientações mais detalhadas sobre como realizar cada etapa da abordagem. Esse detalhamento não será apresentado, devido ao espaço disponível. Vale destacar que as duas abordagens de definição para reutilização foram aplicadas na prática. A abordagem bottom-up foi utilizada para tornar reutilizáveis os processos de uma organização de software (Barreto et al., 2009) e a abordagem top-down foi utilizada para a definição de uma linha de processos de aquisição para uma instituição implementadora de processos (Barreto et al., 2010). 


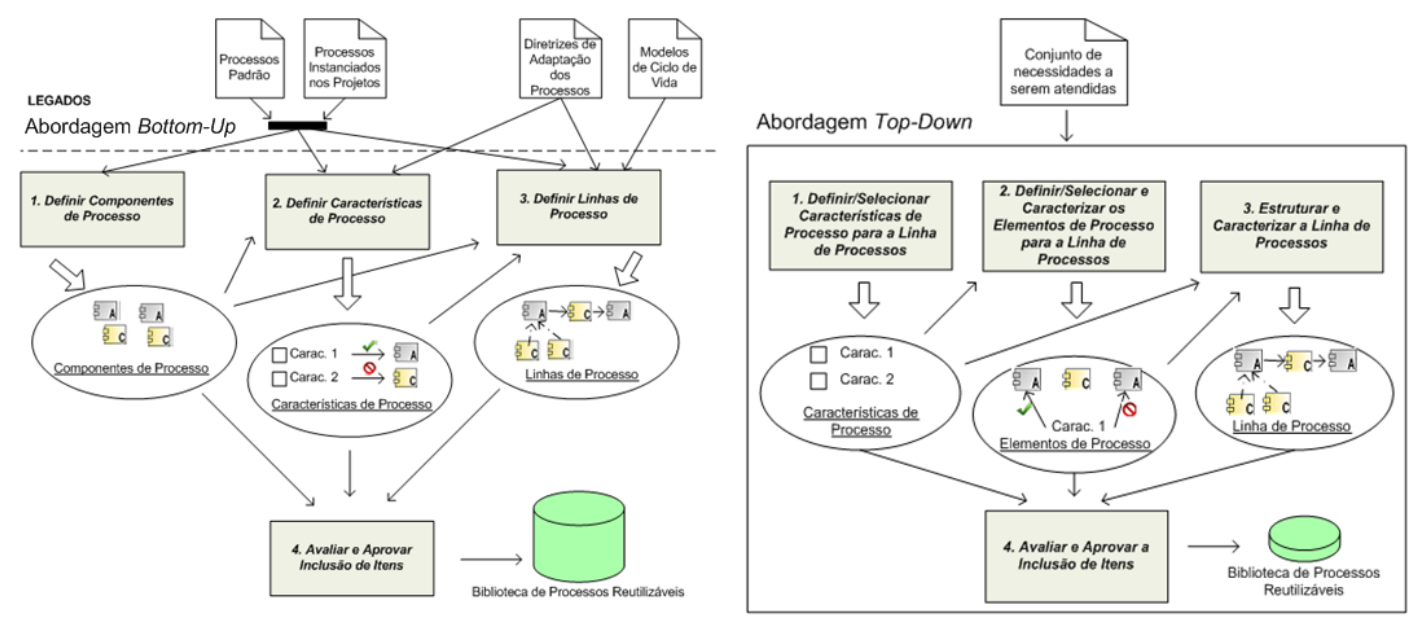

Figura 2. Abordagens para definição de processos para reutilização

\subsection{Definindo Processos com Reutilização}

Neste trabalho, a estratégia para definir processos com reutilização envolve a definição de processos padrão para organizações, a partir de características, componentes e linhas de processo e a definição de processos para projetos, a partir de processos padrão organizacionais, considerando requisitos de alta maturidade.

Um processo padrão organizacional não é necessariamente definido de maneira que possa ser diretamente utilizado por um projeto. Portanto, pode manter algumas possibilidades de variação em aberto, de modo a possibilitar que a decisão sobre como será de fato o processo a ser executado seja tomada na definição de processo para o projeto. Assim, um processo padrão organizacional pode ser visto como uma linha de processos derivada a partir de outra linha mais geral, que é, também, derivada em um processo definido para um projeto. A definição de processos padrão para organizações é composta, nesta tese, por cinco passos principais: (i) Definir informações básicas do processo; (ii) Selecionar características de processo; (iii) Selecionar a linha de processos a ser usada; (iv) Escolher variantes e opcionais; e (v) Solicitar validação do cliente. Ou seja, Nesse caso, a definição se inicia com as informações básicas do processo (seu propósito, requisitos, etc) e com base nisso são selecionadas as características de processo que precisam ser atendidas pelo processo sendo definido. A seleção das características restringe as possibilidades de escolha e guia a definição do processo. Depois é selecionada a linha de processos mais adequada, se houver mais de uma, dentre as que não desrespeitam as características selecionadas. Com a linha escolhida, a estrutura e principais elementos já estarão escolhidos e então devem ser escolhidas variantes para os pontos de variação restantes e deve se decidir sobre os elementos e conexões opcionais. No processo padrão devem ser deixadas algumas possibilidades de variação para serem resolvidas nos projetos. Definido o processo, ele pode ser enviado para validação e uso do cliente.

A outra possibilidade de definição com reutilização é a definição de processos para projetos, conforme ilustrado na Figura 3. Este contexto é o mais afetado na alta maturidade. A definição se inicia com a definição das informações básicas dos processos (requisitos, propósito) e essas informações são utilizadas para escolher qual 
processo padrão deve ser utilizado. Uma vez selecionado o processo padrão, a estrutura principal do processo já estará definida, mas adaptações ainda são necessárias. Assim, é importante estabelecer os critérios que vão guiar a escolha de uma entre várias alternativas possíveis de processo. Vale ressaltar que essa escolha entre alternativas é requisito na alta maturidade. Como critérios podem ser usados os objetivos de qualidade e desempenho de processo para o projeto, existência de medidas, baselines de desempenho, além das próprias características de processo que representam outros critérios importantes. Uma vez definidos os critérios, as alternativas devem ser avaliadas quanto aos critérios e a seleção final das variantes e opcionalidades deve ser feita. Depois, o processo pode ser enviado para avaliação (grupo de qualidade, por exemplo).

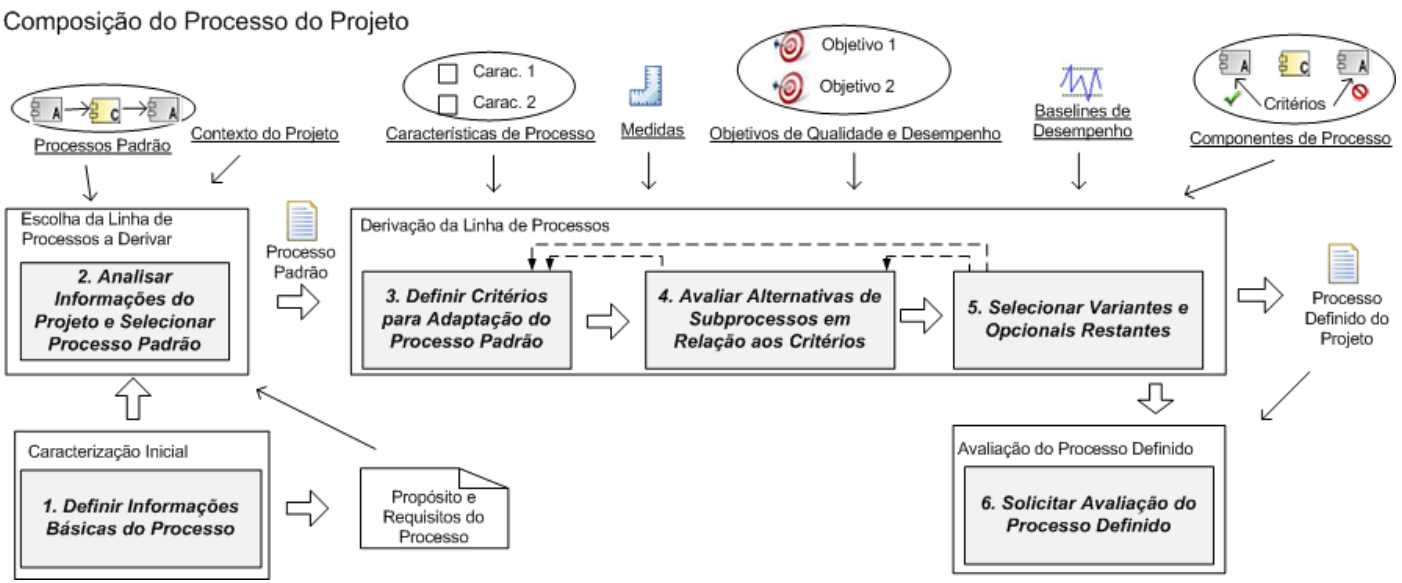

Figura 3. Definição de processo para o projeto a partir de processo padrão

Vale ressaltar que o detalhamento das abordagens fornece orientações mais detalhadas sobre como realizar cada etapa. Esse detalhamento não será apresentado, devido ao espaço disponível. A definição de processos com reutilização foi avaliada em estudo experimental, conforme descrito na seção 4.

\subsection{Ferramentas de Apoio à Abordagem}

Reutilizar processos de maneira sistemática sem a utilização de ferramentas de apoio pode ser uma tarefa muito difícil. Conforme apresentado na seção 3.1, a falta de ferramentas de apoio adequadas foi a dificuldade apontada como sendo a de maior probabilidade de ameaçar a reutilização de processos, dentre as dificuldades consideradas. Assim, tentando possibilitar e facilitar a adoção e prática da reutilização de processos, inclusive considerando a definição de processos em alta maturidade, foi desenvolvido um conjunto de ferramentas de apoio à reutilização de processos.

É importante mencionar que apesar de existirem outras ferramentas de apoio com objetivos semelhantes aos das ferramentas propostas nesta tese, como o Eclipse Process Framework (Eclipse, 2011), o Rational Method Composer (Rational, 2011) e o WebAPSEE (Webapsee, 2011), essas ferramentas não utilizam o conceito de linhas de processo ou de características de processo, além de não forneceram apoio à alta maturidade. Outras ferramentas, como a descrita por Aleixo et al. (2010), apesar de considerarem linhas e características de processo, não apoiam a alta maturidade. 
Para apoiar as estratégias de definição de processos para e com reutilização, apresentadas nas seções anteriores, foi desenvolvido um conjunto de ferramentas de apoio, conforme esquematizado na Figura 4.

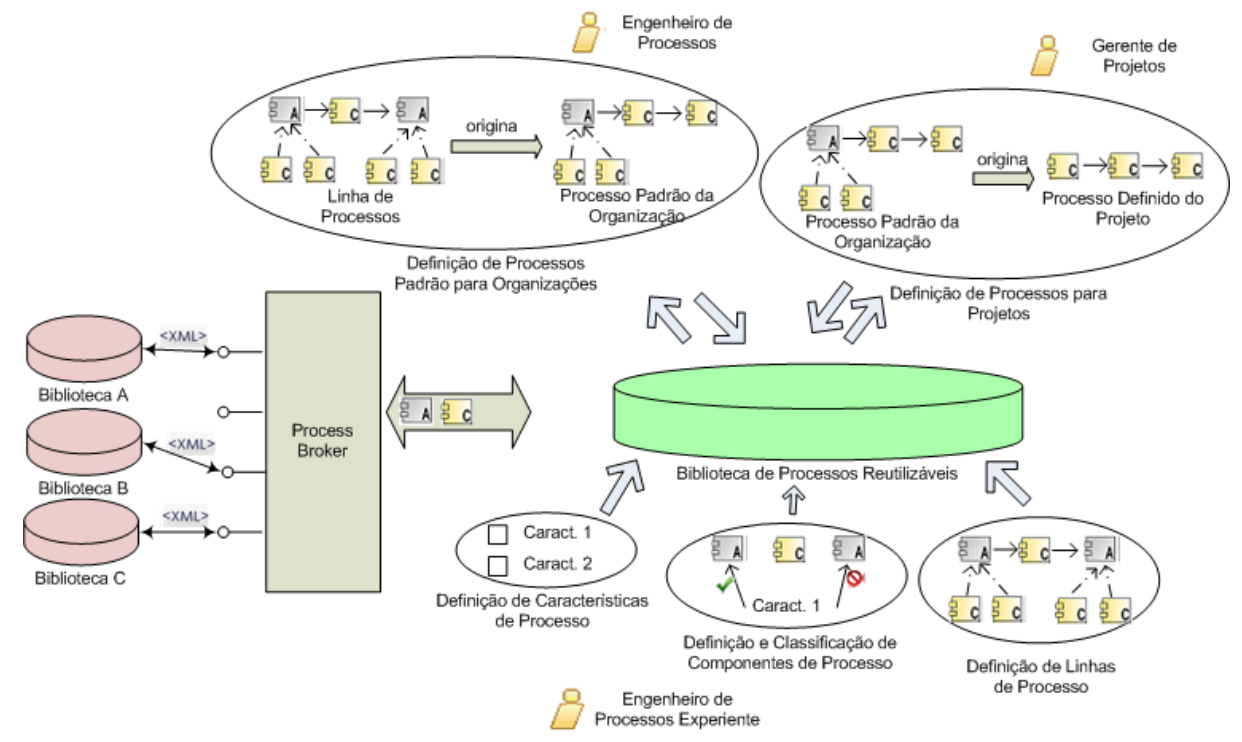

Figura 4. Visão geral das ferramentas de apoio desenvolvidas

$\mathrm{Na}$ parte central da Figura 4 é representada a biblioteca de processos reutilizáveis, que foi desenvolvida utilizando banco de dados Oracle, com uma estrutura baseada na modelagem mencionada na seção 3.1. Abaixo do repositório, são representadas as três principais ferramentas disponíveis para apoiar a estratégia de definição de processos para reutilização, cujos usuários são engenheiros de processo experientes, conforme ilustrado. Na parte superior da Figura 4 são apresentadas as duas ferramentas que apoiam a estratégia de definição de processos com reutilização, cujos usuários são, conforme indicado na Figura 4, engenheiros de processos e gerentes de projetos, respectivamente. O Process Broker (esquerda da Figura 4) permite o intercâmbio de itens reutilizáveis e demais informações relevantes entre bibliotecas de processos reutilizáveis. Foi desenvolvido no contexto de um projeto final de graduação (Vieira e Silva, 2010) que contou com a coorientação do autor deste trabalho.

A Figura 5 exemplifica uma das telas das ferramentas de apoio, ilustrando a derivação de um processo padrão para um projeto. É possível observar, na Figura 5, a estrutura da linha de processos, as decisões possíveis (variantes a selecionar e opcionais a incluir ou não), os critérios selecionados (incluindo quantitativos) e os dados de execução dos processos, para apoiar a escolha de componentes.

\section{Avaliação da Abordagem}

Para avaliar a abordagem proposta neste trabalho e obter mais indícios sobre a viabilidade de sua aplicação e também aprender a partir dessa aplicação, foi planejado e realizado um estudo experimental. Este estudo envolveu: (i) a utilização de componentes, características e linhas de processo; (ii) a utilização da estratégia para definição de processos para projetos, incluindo aspectos de alta maturidade; (iii) a utilização de ferramentas de apoio desenvolvidas. 


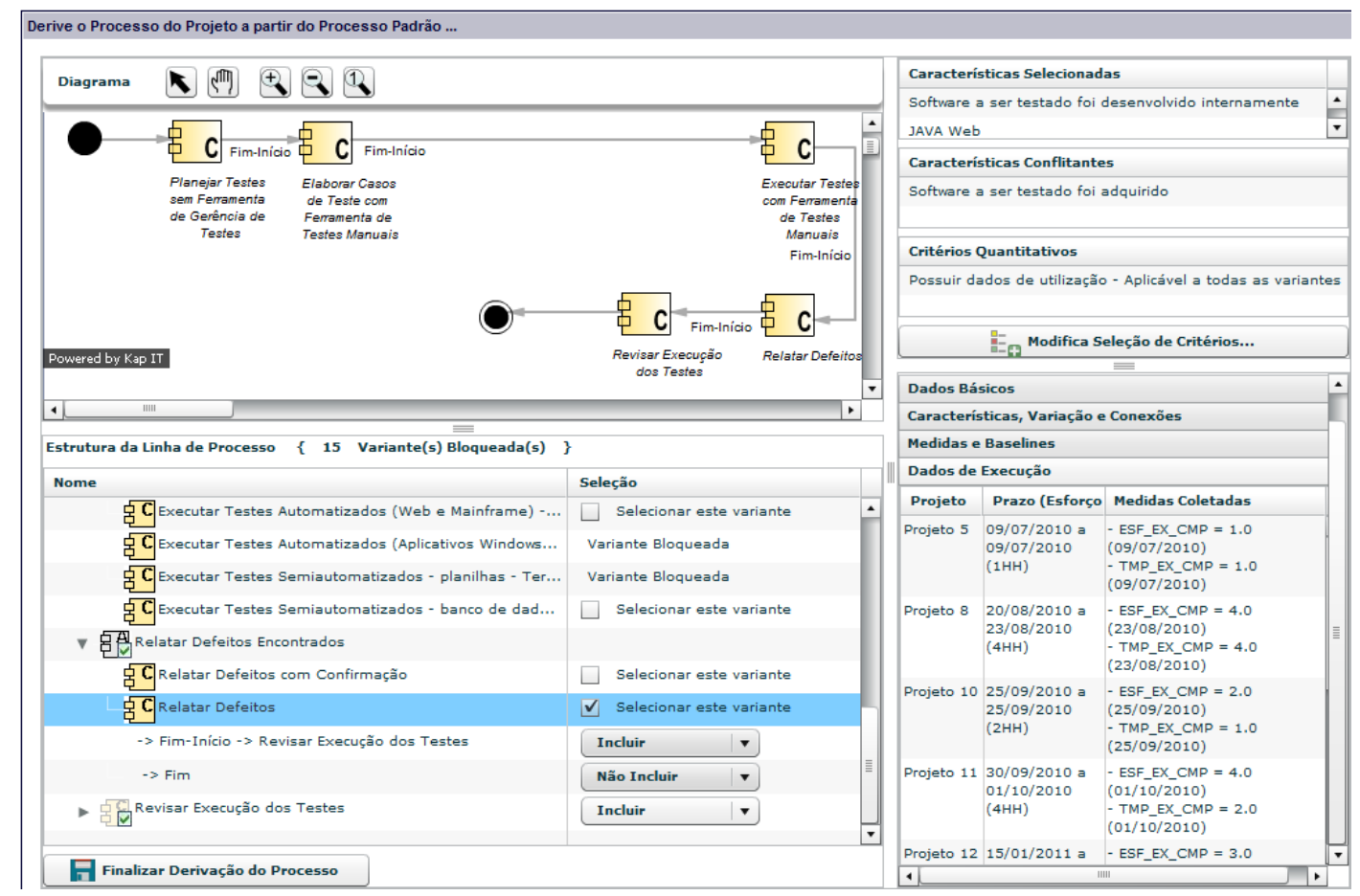

Figura 5. Derivação de processo padrão para definir processo de projeto

O objetivo principal do estudo experimental realizado foi avaliar a utilização de linhas de processos de software na realização da atividade de compor o processo definido para um projeto, considerando aspectos de alta maturidade (objetivos quantitativos de qualidade e desempenho, informações sobre capacidade e estabilidade, medidas). Entretanto, foi possível avaliar, também, um conjunto de outros fatores, como as ferramentas desenvolvidas, as impressões dos participantes ao interagir com a abordagem proposta, entre outros. Foi feita uma comparação entre os resultados da execução da definição de processos para projetos apoiada por uma linha de processos, conforme proposto neste trabalho, e os resultados da execução sem o apoio de linhas de processo, apenas com o apoio de componentes de processo.

O objetivo do estudo experimental realizado foi: Analisar a abordagem proposta para definição de processos baseada em reutilização com a utilização de linhas de processos de software, com o propósito de caracterizar, com respeito à aderência aos requisitos do processo e ao esforço de execução, comparados à execução das mesmas atividades com a utilização de apenas componentes de processo, do ponto de vista do pesquisador, no contexto de engenheiros de processo definindo um processo para um projeto em laboratório.

Foram definidas duas hipóteses nulas para o estudo: $\left(\mathrm{H}_{1}\right)$ o esforço de execução (tempo, em minutos) da atividade sem a utilização de linhas de processo não se altera quando comparado ao tempo de execução da atividade com o apoio de linhas de processo conforme proposto neste trabalho; e $\left(\mathrm{HO}_{2}\right)$ a aderência dos processos definidos (grau de atendimento dos requisitos estabelecidos) para o projeto com a utilização de linhas de processo, conforme proposto neste trabalho, não se altera quando comparada à aderência dos processos definidos para o projeto sem a utilização de linhas de processo. 
Os participantes do estudo deveriam ser engenheiros de software com variados níveis de experiência em definição de processos, desde especialistas até profissionais com muito pouca experiência. A escolha dos participantes foi baseada em princípios não probabilísticos e a população foi determinada por conveniência. Os participantes foram requisitados a realizar a atividade de composição de processo para um projeto de software definido em laboratório, baseado em cenário real (estudo In-vitro). O mesmo projeto e seus requisitos específicos, as mesmas diretrizes de adaptação do processo padrão, os mesmos subprocessos ou elementos de processos e os mesmos dados relacionados à utilização dos processos (medidas, baselines de desempenho, etc) foram apresentados a todos os participantes. O objetivo de cada participante foi compor um processo definido para o projeto descrito, selecionando dentre as alternativas de processo disponíveis (considerando-se os componentes disponibilizados) uma alternativa que atendesse a todos ou ao maior número de requisitos estabelecidos (processo padrão, suas diretrizes de adaptação e requisitos específicos do projeto). Um grupo de participantes deveria realizar a atividade utilizando a ferramenta desenvolvida neste trabalho, com o apoio de linhas de processo (descrevendo as variabilidades do processo, características de processo associadas aos componentes, etc.). Outro grupo de participantes deveria realizar a atividade também utilizando a ferramenta desenvolvida neste trabalho, mas apenas com o apoio dos elementos básicos de processo (componentes de processo e atividades) disponibilizados e funcionalidades de pesquisa desses elementos, que não estariam mapeados para características de processo. Uma vez que se tratava de um cenário com características de alta maturidade, dentre os requisitos estabelecidos foram definidos, também, requisitos de comportamento do processo. Por exemplo, "é desejável que o subprocesso SP2 não apresente esforço de execução superior a 20 homem-hora". E a determinação do atendimento ou não do requisito deveria ser baseada nas informações históricas disponíveis sobre a estabilidade e a capacidade dos subprocessos. $\mathrm{O}$ estudo foi realizado em um período de aproximadamente 3 semanas. Além de 2 participantes em execuções piloto, o estudo contou com outros 21 participantes. Desses, a onze foi atribuído o tratamento com a utilização de linha de processos e aos dez restantes, o tratamento alternativo.

Os resultados do estudo indicaram que sempre houve diferenças significativas (cerca de 25\%) nos desempenhos em relação ao tempo, dependendo do tratamento utilizado. Assim, acredita-se ser possível rejeitar a hipótese $\mathrm{H}_{1}$. Em relação à aderência aos processos, no entanto, nunca houve diferenças significativas nos desempenhos em relação à aderência, independentemente do tratamento utilizado. Portanto, acredita-se não ser possível rejeitar a hipótese $\mathrm{H}_{2}$. Os resultados do estudo foram também analisados em relação à experiência dos participantes e em relação à familiaridade com o cenário utilizado no estudo. Foi possível perceber ao final da análise, que quanto maior o apoio fornecido, menos esforço é necessário para realizar a atividade, como era de se esperar. No entanto, foi surpreendente perceber que o apoio fornecido não influenciou na aderência do processo definido. Uma possível justificativa é que talvez o apoio fornecido mesmo apenas com componentes de processo seja suficiente para garantir boa aderência do processo definido, ainda que com mais esforço. Foi possível perceber, também, que a experiência não influenciou significativamente nos resultados, o que pode ser um indício de que os apoios fornecidos aproximem resultados dos menos experientes dos mais experientes. Assim, com a realização do estudo foi possível identificar indícios de que a abordagem desenvolvida realmente apoia a definição de 
processos considerando requisitos quantitativos, incluindo informações de capacidade e desempenho de subprocessos. Vale salientar, entretanto, que como em qualquer estudo experimental, existem ameaças à validade. Foram analisadas, no estudo realizado, as ameaças à validade interna, externa, de construção e de conclusão, sendo necessários novos estudos para confirmar ou não os resultados observados.

Também foi pedido aos participantes do estudo que respondessem ao mesmo questionário sobre benefícios e dificuldades esperados a partir da utilização de técnicas de reutilização de processos (mencionado na seção 3.1), mas desta vez focando não nas técnicas de forma geral, mas na utilização das técnicas a que foram expostos na realização do experimento. Foi possível perceber, a partir da análise das respostas desse questionário, que a percepção de benefícios melhorou em relação à primeira pesquisa. Ou seja, um nível maior de benefícios foi esperado. Isso pode indicar que o apoio proposto neste trabalho realmente auxilia no alcance dos benefícios analisados. Além disso, percebeu-se que a percepção de dificuldades diminuiu consideravelmente em relação à primeira pesquisa, e a dificuldade "Apoio ferramental inadequado" foi a que teve maior redução. Esse resultado pode indicar que o apoio proposto neste trabalho poderia realmente diminuir o impacto das dificuldades consideradas na pesquisa.

Por fim, foram apresentadas sete afirmações relacionadas à abordagem proposta, para que os participantes indicassem se concordavam ou discordavam e em que grau. As perguntas foram: A abordagem utilizada (i) auxilia a definição de processos; (ii) torna a atividade de definição de processos mais fácil; (iii) permite a reutilização de conhecimento sobre definição de processos; (iv) permite a definição de processos com base na escolha de subprocessos; (v) permite a definição de processos considerando dados de estabilidade e desempenho dos subprocessos, incluindo informações sobre medidas e baselines de desempenho; (vi) permite a definição de processos considerando objetivos quantitativos dos projetos (objetivos quantitativos de qualidade e desempenho de processo); (vii) facilita a seleção dos subprocessos mais adequados para compor um processo em uma dada situação, considerando as diretrizes e requisitos a serem atendidos pelo processo. Para ambos os tratamentos o nível de concordância foi alto, mas aqueles que utilizaram linhas de processo expressaram maior concordância em todas as afirmações. Não houve discordância com nenhuma das afirmações.

Além das avaliações descritas nesta seção (estudo experimental e pesquisa de opinião), foram também realizadas experiências de uso da abordagem no contexto da definição de processos para reutilização, como descrito na seção 3.2 , e outra pesquisa de opinião, conforme descrito na seção 3.1. Com isso, utilizando diferentes tipos de avaliação, todos os cinco requisitos para a abordagem descritos na seção 3 puderam ser avaliados ao longo do desenvolvimento do trabalho.

\section{Conclusão}

Este trabalho apresentou uma abordagem para definição de processos de software baseada em reutilização. A abordagem adapta conceitos normalmente utilizados na reutilização de produtos de software para o contexto da definição de processos, modelando os conceitos envolvidos, estabelecendo como definir processos reutilizáveis e como a partir destes derivar outros processos. Além disso, considera requisitos relacionados à definição de processos em organizações de alta maturidade de processos 
de software. A abordagem também disponibiliza apoio ferramental para realização de suas principais etapas.

As principais contribuições deste trabalho são: (1) A adaptação e a modelagem dos conceitos relevantes da reutilização de produtos de software para o contexto da definição de processos de software, integrados aos demais conceitos relacionados a processos de software e à alta maturidade; (2) O desenvolvimento de uma estratégia para definição de processos para reutilização, a partir de processos de software legados; (3) O desenvolvimento de uma estratégia para definição de processos para reutilização, a partir de um conjunto de requisitos bem definidos; (4) O desenvolvimento de uma estratégia para definição de processos com reutilização, no contexto de instituições implementadoras e organizações de software, para definir processos padrão de organizações; (5) O desenvolvimento de uma estratégia para definição de processos para projetos, com reutilização, considerando requisitos de alta maturidade; (6) O desenvolvimento de ferramental de apoio para a abordagem proposta; (7) Os resultados da pesquisa sobre benefícios e dificuldades esperados com a aplicação de técnicas de reutilização de processos; (8) Os resultados do estudo baseado em revisão sistemática da literatura sobre reutilização de processos de software; (9) Os resultados do estudo experimental sobre a abordagem desenvolvida; (10) Os resultados da experiência de uso de definição de processos para reutilização a partir de processos legados; (11) Os resultados da experiência de uso de definição de linha de processos para aquisição utilizando a abordagem proposta para definição de processos para reutilização.

Durante a execução deste trabalho algumas limitações puderam ser observadas, dentre elas: (1) o fato de que não é possível adaptar um componente de processo para reutilização; (2) alguns aspectos da definição de processos ficaram fora do escopo desta tese, sendo tratados em outros trabalhos; (3) apoio superficial fornecido pelo Process Broker; (iv) pouco apoio fornecido à avaliações dos itens reutilizáveis definidos. Além disso, alguns trabalhos futuros também puderam ser vislumbrados, como a definição de uma abordagem de gerência de configuração de elementos reutilizáveis de processo, o desenvolvimento de mecanismos de junções e diferenças entre componentes de processo, o apoio à execução dos processos com base em componentes e inclusão de mecanismos mais sofisticados para relacionamentos entre características de processo.

\section{Referências}

Acuña, S.T., Antonio, A., Ferré, X., et al., 2000, "The Software Process: Modelling, Evaluation and Improvement". In: CHANG, S.K. (eds), Handbook of Software Engineering and Knowledge Engineering, 1st ed., Singapore, World Scientific Publishing Company.

Aleixo, F.A., Freire, M.A., Santos, W.C., et al., 2010, "Uma Abordagem para Gerência e Customização de Variabilidades em Processos de Software". In: Simpósio Brasileiro de Engenharia de Software, Salvador, Brasil, Outubro.

Barreto, A., Duarte, E., Rocha, A.R., et al., 2010, "Supporting the Definition of Software Processes at Consulting Organizations via Software Process Lines". In: 7th QUATIC, pp. 15-24, Porto, Portugal. 
Barreto, A., Murta, L., Rocha, A.R., 2009, "Componentizando Processos Legados de Software Visando a Reutilização de Processos". In: VIII Simpósio Brasileiro de Qualidade de Software, pp. 189-203, Ouro Preto, Brasil.

Barreto, A., Murta, L., Rocha, A.R., 2011, "Software Process Definition: a Reuse-based Approach", Journal of Universal Computer Science, v. 17, n. 13, pp. 1765-1799.

Card, D.N., Domzalski, K., Davies, G., 2008, "Making Statistics Part of Decision Making in an Engineering Organization", Software, IEEE, v. 25, n. 3, pp. 37-47.

Eclipse, "EPF: Eclipse Process Framework". In: http://www.eclipse.org/epf/, accessed in $15 / 08 / 2011$.

Gary, K.A., Lindquist, T.E., 1999, "Cooperating Process Components". In: International Computer Software and Applications Conference (COMPSAC), pp. 218-223, Phoenix, United States, October.

Iso/Iec-15504, 2004, "Information Technology - Software Process Assessment", Parts 1-9, The International Organization for Standardization and the International Electrotechnical Commission, v. ISO/IEC 15504, Genebra, Suiça.

Montoni, M., 2007, Uma Abordagem para Condução de Iniciativas de Melhoria de Processos de Software, Exame de Qualificação para o Doutorado, Programa de Engenharia de Sistemas e Computação, COPPE/UFRJ, Rio de Janeiro, Brasil.

Osterweil, L., 1987, "Software Processes Are Software Too". In: International Conference on Software Engineering, pp. 2-13, Monterey, Estados Unidos.

Rational, "Rational Method Composer". In: http://www01.ibm.com/software/awdtools/rmc/, accessed in 15/08/2011.

Reis, R.Q., 2002, APSEE-Reuse: Um Meta-Modelo para Apoiar a Reutilização de Processos de Software, Tese de D.Sc., PPGC, UFRGS, Porto Alegre, Brasil.

Rombach, H.D., 2005, "Integrated Software Process and Product Lines". In: International Software Process Workshop, pp. 83-90, Beijing, China, May.

Sei, 2010, CMMI for Development Version 1.3, CMU/SEI-2010-TR-033, Carnegie Mellon University, Software Engineering Institute.

Softex, 2011, " Melhoria de Processo do Software Brasileiro, Guia Geral:2011". In: http://www.softex.br/mpsBr/_guias/default.asp, accessed in 30/07/2011.

Vieira, G.V.M., Silva, W.P.D., 2010, Process Broker: Uma Infra-estrutura de Mediação de Componentes de Processo, Projeto Final de Graduação, Instituto de Computação, Universidade Federal Fluminense, Niterói.

Washizaki, H., 2006, "Building Software Process Line Architectures from Bottom Up". In: Product-Focused Software Process Improvement, pp. 415-421, Amsterdam, Netherlands, June.

Webapsee, "WebAPSEE - Flexible Process Management". In: http://sourceforge.net/projects/webapsee/, accessed in 15/08/2011. 